\title{
Comparison between The Efficacy of Intracoronary Nitroglycerin versus Nitroglycerin Plus Glycoprotein Inhibitors for Treatment of Patients with Thrombolysis in Myocardial Infarction Flow Less Than Three during Primary Percutaneous Coronary Intervention Ahmed Mohamed Kamal Motaweha, Mohamed Ahmed Mosaad Zaki a, Mahmoud Abd El-Naser Ahmed Hussieny ${ }^{\mathrm{b} *}$ \\ ${ }^{a}$ Department of Cardiology, Faculty of Medicine, Al-Azher University ${ }^{b}$ Department of Cardiology, Al- Agoza Hospital \\ *Corresponding author: Mahmoud Abd El-Naser Ahmed Hussieny, Email: mahmoudnaseraswan2108@gmail.com, Mobile: (+20)01121429150
}

\begin{abstract}
Background: Primary percutaneous coronary intervention (PPCI) is currently the cornerstone of management in patients with ST-segment elevation myocardial infarction (STEMI).

Aim of the work: comparison between the efficacies of intracoronary nitroglycerin (NTG) versus nitroglycerin plus glycoprotein inhibitors (GP) IIb/IIIa for treatment of patients with thrombolysis in myocardial infarction flow less than three during primary percutaneous coronary intervention.

Patients and methods: we prospectively enrolled 30 patients with STEMI undergoing PPCI randomized to receive IC NTG only $(n=15)$ versus NTG plus GP IIb/IIIa inhibitors $(n=15)$ during PPCI. The primary outcome was the incidence of angiographic MVO as defined by thrombolysis in myocardial infarction flow less than 3 or thrombolysis in myocardial infarction flow 3 with myocardial blush grade less than 2.

Results: NTG plus GP IIb/IIIa inhibitors were associated with better primary outcome compared to NTG only TIMI 3 $\& \mathrm{MBG}>=2$ (40\%vs $73.3 \%$, respectively; $\mathbf{P}=0.036$ ). There was a trend towards improved left ventricular ejection fraction with NTG plus GP IIb/IIIa inhibitors (44.33 \pm 9.76 vs $52.87 \pm 11.23$, respectively; $\mathbf{P}=0.035)$. In addition, NTG plus GP IIb/IIIa inhibitors was associated with lower incidence of 30-day major adverse cardiovascular events (26.7 vs. $66.7 \%$ respectively; $\mathrm{P}=0.028$ ).

Conclusion: In PPCI, NTG plus GP IIb/IIIa inhibitors resulted in significant improvements in no-reflow and MVO with a better safety profile compared to NTG only. Larger trials should be conducted to confirm these results.

Keywords: Acute myocardial infarction, Microvascular obstruction, Primary percutaneous coronary intervention, No reflow, Nitroglycerin, Platelets glycoprotein IIb/IIIa inhibitors.
\end{abstract}

\section{INTRODUCTION}

Primary percutaneous coronary intervention (PPCI) is currently the cornerstone of management in patients with ST-segment elevation myocardial infarction (STEMI) ${ }^{(1)}$.

Despite successful recanalization by angioplasty, $30-50 \%$ of the patients have poor outcomes because of microvascular obstruction (MVO) ${ }^{(2,3)}$. The two main indicators of MVO are Thrombolysis in Myocardial Infarction (TIMI) flow and myocardial blush grade (MBG) ${ }^{(4,5)}$. $\mathrm{MBG}$ is a more sensitive indicator compared to TIMI flow, and some patients will show reduced myocardial reperfusion by MBG even with achievement of TIMI 3 flow ${ }^{(5)}$. Potential mechanisms of MVO include vasospasm, distal embolization of thrombus or debris, oxygen free radical-mediated endothelial injury (ischemic-reperfusion injury), capillary plugging by erythrocytes and neutrophils, and intracellular/interstitial edema ${ }^{(6,7)}$.

Some medications have been shown to be effective in the management of NR and MVO. Among these NTG and GP IIb/IIIa.NTG is a nitric oxide donor that results in vasodilation of vascular smooth muscles by increasing the formation of cGMP through guanylate cyclase stimulation. GP IIb/IIIa antagonizes $\alpha \operatorname{IIb} \beta 3$ selectively and competitively to inhibit platelet aggregation. $\alpha \operatorname{IIb} \beta 3$ engagement is required for aggregation through all pathways, blocking this receptor inhibits platelet aggregation more effectively (8).

Unfortunately, despite these potential pharmacological benefits, studies directly comparing these NTG and GP IIb/IIIa.NTG for the prevention of NR and MVO during PPCI are not available and hence this is still a topic of debate ${ }^{(9)}$. We sought to compare the safety and efficacy of these two medications in the prevention of this life-threatening complication.

\section{AIM OF THE WORK}

Comparison between the efficacy of intracoronary nitroglycerin (NTG) versus nitroglycerin plus glycoprotein inhibitors (GP)IIb/IIIa for treatment of patients with Thrombolysis In Myocardial Infarction flow less than three during primary percutaneous coronary intervention.

\section{PATIENTS AND METHODS}

\section{Study population}

This prospective observational study that was conducted from May 2018 to February 2019 and included 30 patients who presented to the Emergency 
Department of Sayed Galal Hospital with acute ST elevation myocardial infarction and were eligible for PPCI. Informed consents for enrollment in the study were obtained after discussing all potential benefits and possible risks of the study protocol. As this is the first head-to-head comparison study for these medications in PPCI, the sample size was intended to compare the safety and efficacy of either protocols for the prevention of NR and MVO in PPCI.

Approval of the Ethical Committee: An approval of the study was obtained from Al-Azhar University Academic and Ethical Committee.

\section{Exclusion criteria:}

(i) Culprit lesion in the left main coronary artery or a by-pass graft,

(ii) Severe renal insufficiency on hemodialysis,

(iii) A history of previous myocardial infarction,

(iv) Previous hemorrhagic stroke and

(v) Age above 70 years.

Thirty patients were randomized to receive intracoronary (IC) NTG $(n=15)$ group I versus IC NTG plus GP IIb/IIIa inhibitors ( $n=15)$ group II. Generation of treatment assignment was performed after informed consent was obtained, through concealed envelopes opened by a trainee, so that a $1: 1$ randomization ratio was achieved. The nursing personnel were then asked to prepare the study drugs. Both opening of the envelope and drug preparation were performed after diagnostic angiography to establish eligibility.

In group I, NTG was $(200 \mu \mathrm{g} / \mathrm{mL})$ was manually infused through guiding catheters for 1 minute and an angiography was done 1 minute later. The bolus drug dose was $200 \mu \mathrm{g}$ for nitroglycerin. If the coronary flow was not satisfactory, another bolus of the same dose was given until reaching the maximum dose of nitroglycerin $1000 \mu \mathrm{g}$. Before and after drug infusion, heart rate and blood pressure were recorded for drug safety evaluation (10).

Patients in group II with TIMI flow below 3 and myocardial blush grade below 3 , received intracoronary bolus administration of tirofiban $(10 \mu \mathrm{g} / \mathrm{kg})$ followed by intravenous tirofiban infusion $(0.15 \mu \mathrm{g} / \mathrm{kg} / \mathrm{min})$ for $18 \mathrm{hr}$. Then, $100 \mathrm{mcg}$ intracoronary nitroglycerin. The dose was slowly and gradually increased up to $400 \mathrm{mcg}$ and TIMI flow grade and MBG were re-evaluated then the results were recorded (11). Otherwise, balloon predilation was allowed before drug administration. All patients received aspirin $325 \mathrm{mg}$ chewable, clopidogrel $300 \mathrm{mg}$ loading dose, and atorvastatin $80 \mathrm{mg}$ before the index procedure. Adjunctive therapies such as thrombus aspiration was prohibited as a part of the protocol to avoid confounding factors that might affect the outcomes. After angioplasty, all patients were admitted to the coronary care unit, where the conventional antiischemic therapies were continued.

\section{Primary outcome}

The primary outcome was the incidence of angiographic MVO. This was defined as final epicardial coronary TIMI flow less than 3, or TIMI flow 3 with an MBG less than 2 in the infarct-related artery ${ }^{(4,5)}$. Coronary angiograms were analyzed by two independent operators, who were blinded to the randomization and study medication administered, to assess the culprit lesions characteristics ${ }^{(12)}$, TIMI flow grade ${ }^{(6)}$ and MBG ${ }^{(13)}$.

\section{Secondary outcomes}

The secondary outcomes included the incidence of STR at least $70 \%$, left ventricular ejection fraction (LVEF) and wall motion score index (WMSI) by twodimensional echocardiography (Vivid S5; GE Healthcare, Little Chalfont, UK) as well as major adverse cardiovascular events (MACE).

LVEF and WMSI were assessed at 3-5 days after PPCI. LVEF was calculated using the biplane Simpson method. The WMSI was defined as the sum of the scores of all segments divided by the total number of segments. Individual scores were calculated as follows: normokinesis $=1 ;$ hypokinesis $=2$; akinesis $=3$; dyskinesis $=4$; and aneurysm $=5$.

The MACE was assessed at 30-day after PPCI and was defined as a composite of cardiovascular death, nonfatal myocardial infarction, target vessel revascularization, or recurrent hospitalization secondary to heart failure.

Safety outcomes included incidence of procedural hypotension (defined as systolic blood pressure < 90 $\mathrm{mmHg}$ ) and incidence bleeding as hematemesis, hemoptysis, melena or uncontrolled bleeding from puncture site.

\section{Statistical analysis}

Statistical analysis was carried out using SPSS 16 for Windows (SPSS Inc., Chicago, Illinois, USA). Continuous variables were expressed as the mean $\pm \mathrm{SD}$, whereas discrete variables were presented as absolute values, percentages, or both. Continuous variables were compared using Student's t-test. Discrete variables were compared using the $\chi 2$-test. A $\mathbf{P}$-value of less than or equal to 0.05 was considered significant.

\section{RESULTS}

Both groups were comparable in terms of the baseline clinical and angiographic characteristics. Sixty-Five percent of the population were men, and the left anterior descending artery was the most common infarct-related artery $(66.6 \%)$ among both groups. The baseline clinical and angiographic characteristics are summarized in table (1). 


\section{Procedural data}

The stent characteristics and the percentage of balloon angioplasty before and after stenting were balanced between both groups. The IC GP IIb/IIIa inhibitors plus NTG group achieved better Thrombolysis In Myocardial Infarction (TIMI)flow 3 and MBG was also improved compared to NTG (11(73\%) vs 6(40\%)), respectively; $(P=0.036)$. The procedural data are summarized in table (2).

\section{Primary outcome}

The incidence of angiographic MVO was lower in the IC GP IIb/IIIa inhibitors plus NTG group compared with the NTG group (26.7 vs. $46,6 \%$, respectively; $\mathbf{P}=$ 0.438) (Fig1). In the IC GP IIb/IIIa inhibitors plus NTG group, $20 \%$ of the patients had TIMI flow less than 3 and $6.7 \%$ had TIMI 3 flow with MBG less than 2 . However, $33.3 \%$ of the patients in the NTG only group had TIMI flow less than 3 and $26.7 \%$ had TIMI 3 flow with MBG less than 2. As shown in table (3).

\section{Secondary outcomes}

The results which were obtained from follow up patients post $\mathrm{PCI}$ by $2 \mathrm{D}$ echocardiography showed that wall motion score index (WMSI) was $1.54 \pm 0.30$ (range 1.125-2.06) in group I and in group II was $1.27 \pm 0.39$ (range 1-2.5) $(\mathrm{P}$-value=0.043). The Ejection Fraction (EF) was $44.33 \pm 9.76$ (range 28-57) in group I. On the other hand EF in Group II was $52.87 \pm 11.23$ (range 2971) $)(\mathrm{P}$-value $=0.035)$ there was statistically significant difference between groups according to Echo (Table 3).

One month later, clinical follow-up was obtained in 30 patients.

$66.7 \%$ (10) patients' events were; one death, two non-fatal MIs and 7 readmission with ACS symptoms in group I. On the other hand in combination therapy group II, $26.7 \%$ (4) patient were; one death, zero nonfatal MIs, 3 readmissions ACS and 3 HF. statistically significant difference between groups according to MACE (P-value=0.028) (Table 3 \& fig 2).

Table (1): Comparison between study groups according to demographic data, risk factors, type of STEMI and culprit vessel

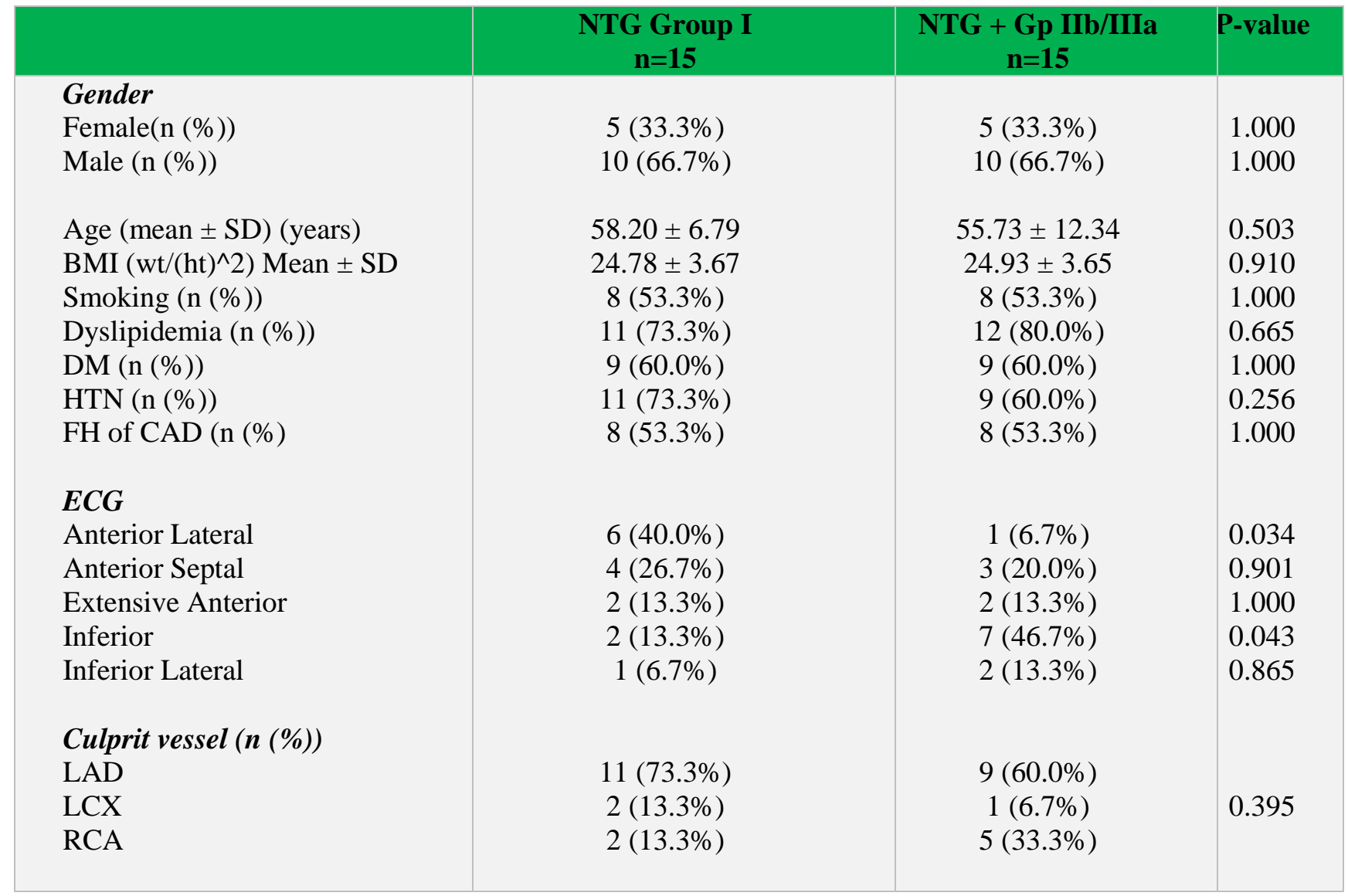

CAD, coronary artery disease; DM, diabetes mellitus; FH, family history; HTN, hypertension; LAD, left anterior descending; LCX, left circumflex; PCI, percutaneous coronary intervention; RCA, right coronary artery; NTG nitroglycerin; GP glycoprotein. A Culprit lesions were classified according to the ACC/AHA criteria (12). 
Table (2): Comparison between groups according to procedural data

\begin{tabular}{|c|c|c|c|}
\hline Procedural Data Post Echo & $\begin{array}{c}\text { Group I: NG } \\
(n=15)\end{array}$ & $\begin{array}{c}\text { Group II: } \\
\text { NG + GP IIb, IIIa } \\
(n=15)\end{array}$ & p-value \\
\hline $\begin{array}{l}\text { Stent } \\
\text { No } \\
\text { Yes }\end{array}$ & $\begin{array}{c}0(0.0 \%) \\
15(100.0 \%)\end{array}$ & $\begin{array}{c}2(13.3 \%) \\
13(86.7 \%)\end{array}$ & 0.143 \\
\hline $\begin{array}{l}\text { Number of stents } \\
0 \\
1 \\
2 \\
3 \\
\end{array}$ & $\begin{array}{c}0(0.0 \%) \\
12(80.0 \%) \\
3(20.0 \%) \\
0(0.0 \%)\end{array}$ & $\begin{array}{c}2(13.3 \%) \\
9(60.0 \%) \\
3(20.0 \%) \\
1(6.7 \%)\end{array}$ & 0.330 \\
\hline $\begin{array}{l}\text { Balloon Pre dilatation } \\
\text { No } \\
\text { Yes }\end{array}$ & $\begin{array}{c}0(0.0 \%) \\
15(100.0 \%)\end{array}$ & $\begin{array}{c}2(13.3 \%) \\
13(86.7 \%)\end{array}$ & 0.143 \\
\hline $\begin{array}{l}\text { Post Dilatation } \\
\text { No } \\
\text { Yes }\end{array}$ & $\begin{array}{c}0(0.0 \%) \\
15(100.0 \%)\end{array}$ & $\begin{array}{c}2(13.3 \%) \\
13(86.7 \%)\end{array}$ & 0.143 \\
\hline $\begin{array}{l}\text { Aspiration Catheter } \\
\text { No } \\
\text { Yes }\end{array}$ & $\begin{array}{c}15(100.0 \%) \\
0(0.0 \%)\end{array}$ & $\begin{array}{c}14(93.3 \%) \\
1(6.7 \%) \\
\end{array}$ & 0.309 \\
\hline
\end{tabular}

Table (3): Efficacy and safety outcomes

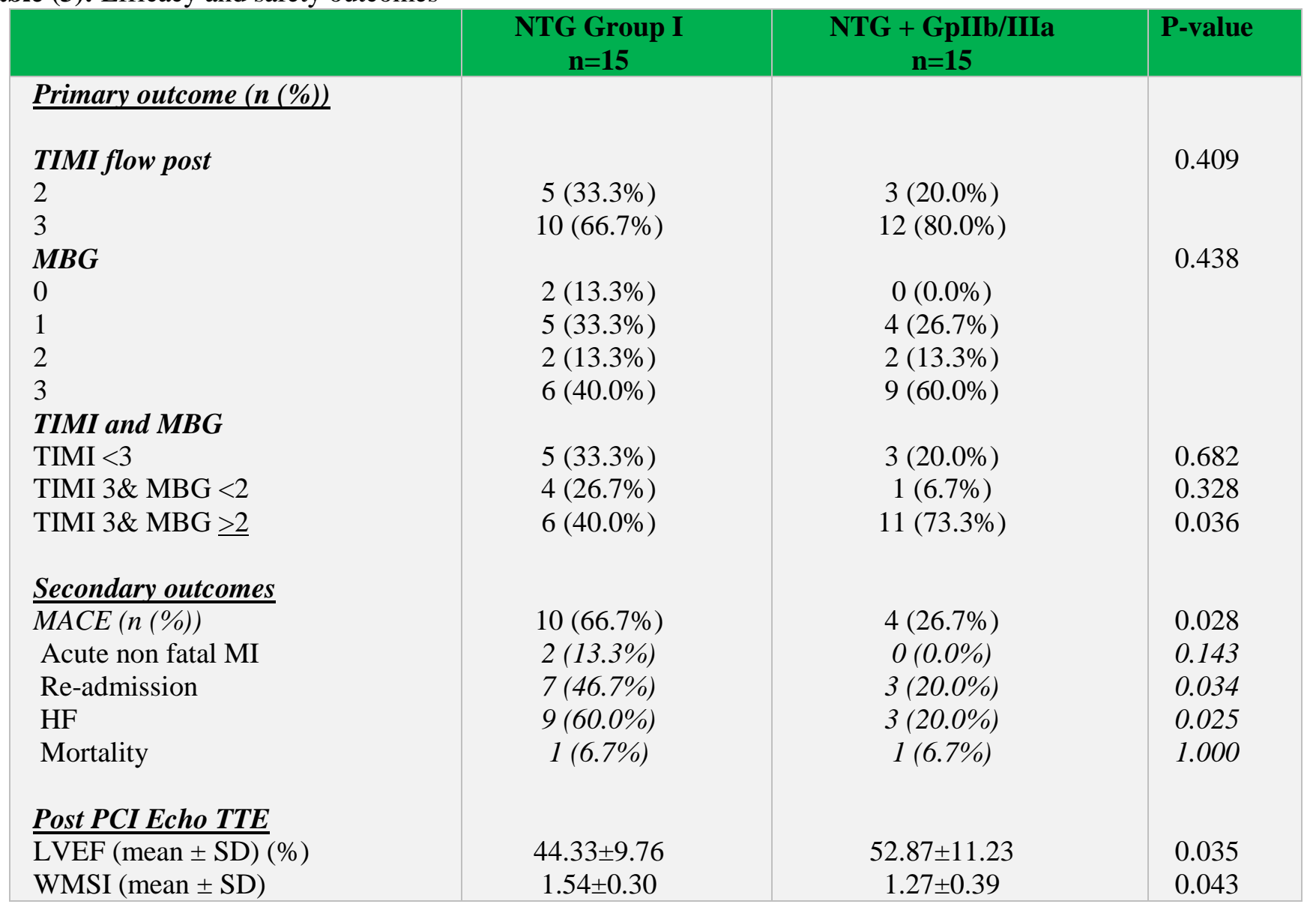

ACS, Acute Coronary Syndrome; HF, heart failure; LVEF, left ventricle ejection fraction; MACE, major adverse cardiovascular events; MBG, myocardial blush grade; MI, myocardial infarction; MVO, microvascular obstruction; PCI, percutaneous coronary intervention; NTG Nitroglycerin; TIMI, Thrombolysis In Myocardial Infarction; WMSI, wall motion score index. 


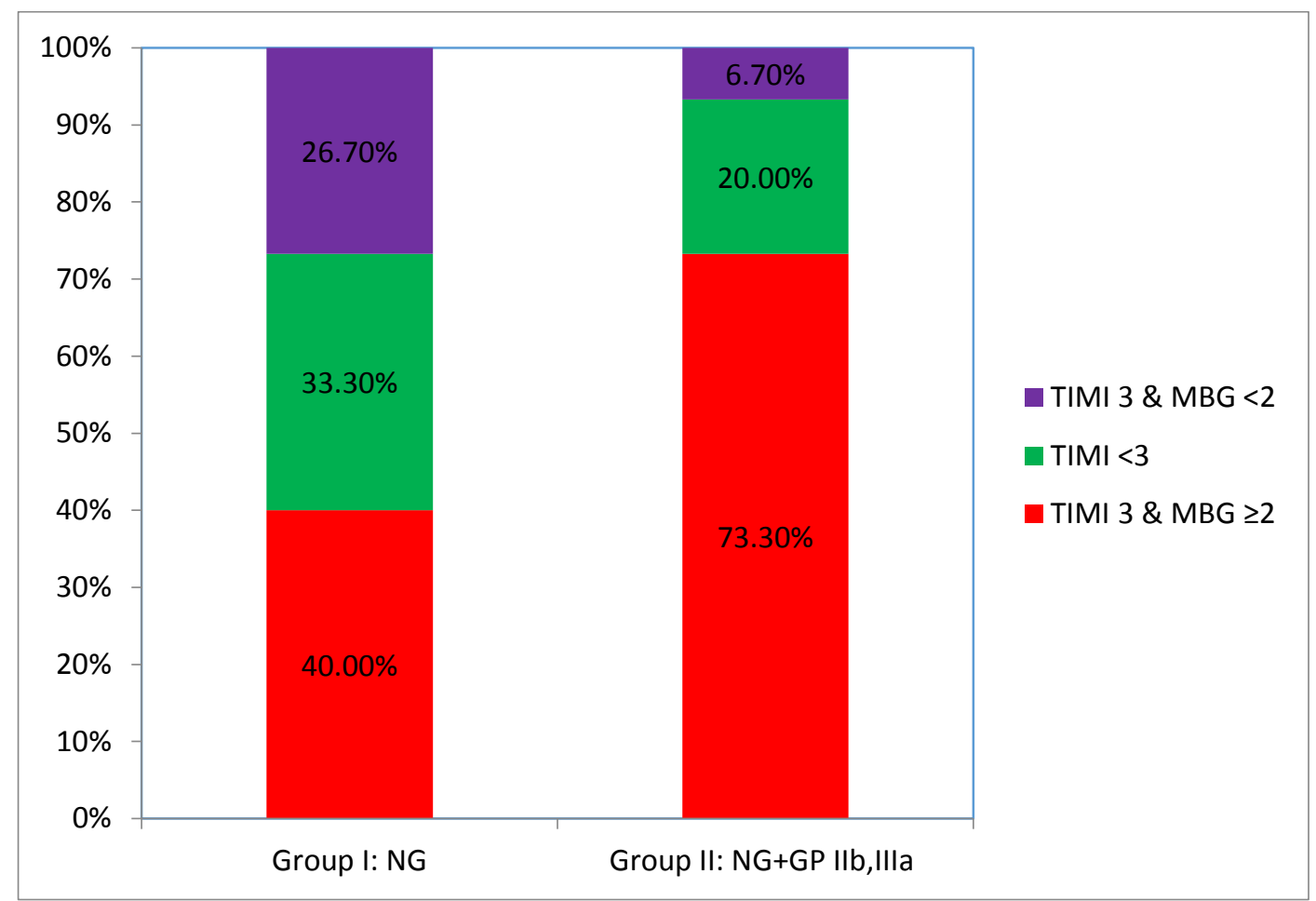

Fig. (1): Bar chart between groups according to TIMI and MBG.

GP, glycoprotein; MBG, myocardial blush grade; MVO, microvascular obstruction; NTG nitroglycerin; TIMI, Thrombolysis In Myocardial Infarction.

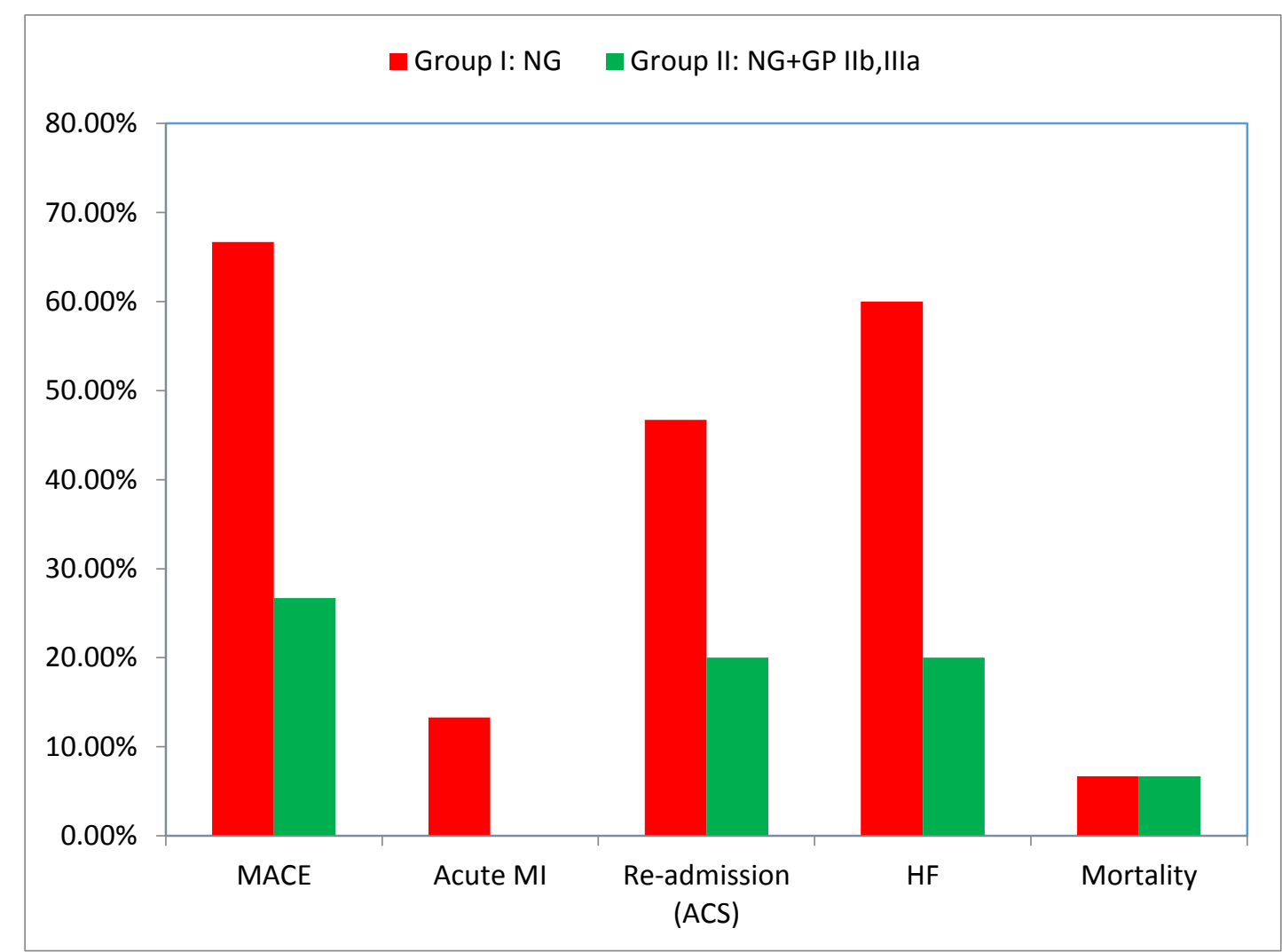

Fig (2): Bar chart between groups according to acute MI, Re-admission, HF, mortality and MACE.

ACS, Acute Coronary Syndrome; HF, heart failure; LVEF, left ventricle ejection fraction; MACE, major adversecardiovascular events; MI, myocardial infarction; MVO, microvascular obstruction; PCI, percutaneous coronary intervention; NTG, Nitroglycerin. 


\section{DISCUSSION}

In this prospective single-center randomized study of 30 patients presenting with STEMI, we compared the efficacy and safety of IC NTG versus NTG plus GP IIb/IIIa inhibitors in MVO and NR. IC combination therapy NTG \& GP IIa/IIIb inhibitors was associated with less angiographic MVO, better LVEF at 3-5 days after PPCI, trend toward better STR and a trend toward better LVEF at 3-5 days after PPCI compared to NTG only. This indicated a potential role in improving reperfusion and outcomes after relief of the coronary obstruction in patients with STEMI. There was improvement in MACE at 30 days better than in NTG group only.

Improvement of outcomes after PPCI is crucial and research is ongoing to determine the optimal drugs for anticoagulation ${ }^{(14)}$, the role of adjunctive interventions such as aspiration thrombectomy ${ }^{(15,16)}$, appropriate timing for complete revascularization ${ }^{(17},{ }^{18)}$ aiming at better myocardial perfusion after revascularization of the culprit artery and improving survival. MVO after PPCI is a complication that leads to worse outcomes and, unfortunately, the proper prevention protocol of this phenomenon is not well established ${ }^{(7,10)}$.

Angiographic evaluation of our study during PCI showed that the incidence of TIMI flow less than 3 in group I was $33.3 \%$ (5 patients) and TIMI $3 \&$ MBG $>=2$ was $40 \%$ (6 patient). However, $20 \%$ (3 patient) had TIMI flow less than 3 and $73 \%$ (11 patients) had TIMI $3 \& \mathrm{MBG}>=2$ in group II with statistically significant difference between both groups (P-value $=0.036$ ). There is different studies were applied to achieve best angiographic results as the pathogenesis of no-reflow is complex. Several known causes including embolization, ischemia-reperfusion injury and individual predisposition of coronary microcirculation to injury ${ }^{(19)}$ are variable in different patients. Current several monotherapies are reported to have certain effects, but not satisfactory at all.

There are convincing randomized trial data for hard clinical outcome using GP II b/IIIa inhibitors in the setting of ST elevation MI ${ }^{(20)}$ and its use in this setting is a class II indication in the current 2011 PCI guidelines ${ }^{(21)}$. It is possible that one mechanism of benefit is the reduction in the no-reflow phenomenon in the GP IIb/IIIa treated patients. Large scaled clinical researches have proven the preventive effects of platelet membrane glycoprotein $\mathrm{II} \mathrm{b} / \mathrm{III}$ a receptor antagonists on the no-reflow events after AMI.

The results of Zhou et al. ${ }^{(22)}$ study showed that there was a reduction in combination therapy group by about $62.9 \%$ in the incidence of no-reflow after primary PCI in patients with acute myocardial infarction compared to control group in high-risk patients, which agrees with our study. In addition, Zhou et al. ${ }^{\text {(22) }}$ study showed that LVEF \% was $53 \pm 8$ in high risk group treated with combination therapy and $44 \pm 6$ in high-risk control not treated by combination therapy with statistically significant difference between both groups according to Echo. These results are in concordance with our results

As regards the study of Zhou et al. (22) combination therapy for no-reflow in patients with AMI after PCI, they did not use single definite period (30 days as our study). They used three different periods to reassess patients for MACE. In hospital stay $(n=621)$ period, there were $6.5 \%$ (10 patients) complaining from MACE in control group and 3.8\% (4 patients) in combination therapy group. The second period was 3 months after PPPCI $(n=583)$. There were $14.4 \%(14$ patients) in control group and $8.1 \%$ (8 patients) in combination therapy group re-admitted due to MACE. The last period was 6 months $(\mathrm{n}=552)$ post-PPCI, $26.4 \%$ (24 patients) in control group and $12.5 \%$ (12 patients) in combination therapy group readmitted due to MACE.

Zhou et al. ${ }^{(22)}$ was large study and they used long duration time follow up. On the other hand, our study was small-size sample and follow up for 30 days only but the end-point results showed that tirofiban as a part of combination therapy had better outcome than monotherapy in PPCI regarded to MACE.

\section{CONCLUSION}

Our study showed that combination of intracoronary administration of platelet membrane glycoprotein II b/IIIa receptor antagonist (tirofiban) in addition to nitroglycerin may restore coronary flow in patients with acute myocardial infarction who developed no-reflow phenomenon after percutaneous coronary balloon intervention and prevent microvascular obstruction. Clinical outcomes was better with this combination as LVEF was higher and MACE rate were lower than the other group that was treated by nitroglycerin only.

\section{RECOMMENDATION}

From this study, we recommend that patients with acute STEMI undergoing PPCI and developed noreflow or slow flow should be treated by administration intracoronary combination of platelet membrane glycoprotein $\mathrm{II} \mathrm{b} / \mathrm{III}$ a receptor antagonist (tirofiban) in addition to nitroglycerin to restore coronary flow and decrease MVO incidence in these patients. We also recommend in future using this combination in patients with high risk of no-reflow (no-flow score $\geq 10$, by using a noflow risk prediction model) to reduces the incidence of no-reflow after primary PCI in patients with acute myocardial infarction.

\section{REFERENCES}

1. Weaver WD, Simes RJ, Betriu A et al. (1997): Comparison of primary coronary angioplasty and 
intravenous thrombolytic therapy for acute myocardial infarction: a quantitative review. JAMA., 278: 20932098.

2. Ito H (2006): No-reflow phenomenon and prognosis in patients with acute myocardial infarction. Nat Clin Pract Cardiovasc Med., 3:4 99-506.

3. Morishima I, Sone T, Okumura K et al. (2000): Angiographic no-reflow phenomenon as a predictor of adverse long-term outcome in patients treated with percutaneous transluminal coronary angioplasty for first acute myocardial infarction. J Am Coll Cardiol., 36: 1202-1209.

4. Niccoli G, Rigattieri S, de Vita MR et al. (2013): Openlabel, randomized, placebo-controlled evaluation of intracoronary adenosine or nitroprusside after thrombus aspiration during primary percutaneous coronary intervention for the prevention of microvascular obstruction in acute myocardial infarction: the REOPEN-AMI study (Intracoronary Nitroprusside Versus Adenosine in Acute Myocardial Infarction). JACC Cardiovasc Interv., 6: 580-589.

5. Henriques JPS, Zijlstra F, van't Hof AWJ et al. (2003): Angiographic assessment of reperfusion in acute myocardial infarction by myocardial blush grade. Circulation, 107: 2115-2119.

6. Kloner RA (1989): No reflow revisited. J Am Coll Cardiol., 14: 1814-1815

7. Erbel $R$ and Heusch $G$ (2000): Coronary microembolization. J Am Coll Cardiol., 36: 22-24.

8. Armstrong PW, Gershlick AH, Goldstein $\mathbf{P}$ et al. (2013): STZREAM Investigative Team. Fibrinolysis or primary PCI in ST-segment elevation myocardial infarction. N Engl J Med., 368: 1379-1387.

9. Singh BN, Ellrodt G and Peter CT (1978): Verapamil: a review of its pharmacological properties and therapeutic use. Drugs, 15: 169-197.

10. Fernandes M, Fish R, Canales J (2012): Restoration of Microcirculatory Patency after Myocardial Infarction: Results of Current Coronary Interventional Strategies and Techniques. Texas Heart Institute Journal, 39 (3): 342-350.

11. 11Hansen PR, Iversen A, Abdulla J (2010): Improved clinical outcomes with intracoronary compared to intravenous abciximab in patients with acute coronary syndromes undergoing percutaneous coronary intervention: a systematic review and metaanalysis. J Invasive Cardiol., 22: 278-82.

12. Ryan TJ, Faxon DP, Gunnar RM et al. (1988): Guidelines for percutaneous transluminal coronary angioplasty. A report of the American College of Cardiology/American Heart Association Task Force on Assessment of Diagnostic and Therapeutic Cardiovascular Procedures (Subcommittee on
Percutaneous Transluminal Coronary Angioplasty). Circulation, 78: 486-502.

13. Van't Hof AW, Liem A, Suryapranata H et al. (1998): Angiographic assessment of myocardial reperfusion in patients treated with primary angioplasty for acute myocardial infarction: myocardial blush grade. Zwolle Myocardial Infarction Study Group. Circulation, 97: 2302-2306

14. Mahmoud A, Saad M, Elgendy AY et al. (2015): Bivalirudin in percutaneous coronary intervention, is it the anticoagulant of choice? Cardiovasc Ther., 33: 227235.

15. Jolly SS, Cairns JA, Yusuf S et al. (2015): Randomized trial of primary PCI with or without routine manual thrombectomy. N Engl J Med., 372: 1389-1398.

16. Elgendy IY, Huo T, Bhatt DL et al. (2015): Is aspiration thrombectomy beneficial in patients undergoing primary percutaneous coronary intervention? Metaanalysis of randomized trials. Circ Cardiovasc Interv., 8: e002258.

17. Elgendy IY, Wen X, Mahmoud A et al. (2015): Complete versus culprit-only revascularization for patients with multi-vessel disease undergoing primary percutaneous coronary intervention: an updated metaanalysis

of randomized trials. Catheter Cardiovasc Interv., 88 (4): 501-505.

18. Saad M, Rashed A, El-Kilany W et al. (2016): Preliminary report on the safety and efficacy of staged versus complete revascularization in patients with multivessel disease at the time of primary percutaneous coronary intervention. Int J Angiol., 26 (3): 143-147.

19. Niccoli G, Burzotta F, Galiuto L (2009): Myocardial no-reflow

humans. J Am Coll Cardiol., 54: 281-292

20. Montalescot, Antoniucci, Kastrati, Neumann (2007): Abciximab primary coronary stenting of ST-elevation myocardial infarction. a European meta-analysis on individual patients' data with longterm follow-up. European Heart Journal, 28 (4): 443-449.

21. Levine GN, Bates ER, Blankenship JC (2011): 2011 ACCF/AHA/SCAI Guideline for Percutaneous Coronary Intervention: A Report of the American College of Cardiology Foundation/American Heart Association Task Force on Practice Guidelines and the Society for Cardiovascular Angiography and Interventions. Circulation, 124: 574-651.

22. Zhou SS, Tian F, Chen YD et al. (2015): Combination therapy reduces the incidence of no-reflow after primary percutaneous coronary intervention in patients with ST segment elevation acute myocardial infarction. Journal of Geriatric Cardiology, 12: 135-142 